CONTRIBUCIÓN DEL $3^{\text {ER }}$ CONGRESO LATINOAMERICANO DE MACROINVERTEBRADOS DE AGUA DULCE: BIODIVERSIDAD Y ECOLOGÍA FUNCIONAL EN EL NEOTRÓPICO

\title{
MACROINVERTEBRADOS ACUÁTICOS DE LA CIÉNAGA DE SAHAYA Y EN TRES DE SUS AFLUENTES (CESAR, COLOMBIA)
}

\author{
AQUATIC MACROINVERTEBRATES FROM SAHAYA WETLAND AND THREE OF ITS \\ TRIBUTARIES (CESAR, COLOMBIA)
}

José Luis Hernández, Cristian J. Guzmán-Soto y César E. Tamaris-Turizo

\begin{abstract}
RESUMEN
Los macroinvertebrados acuáticos representan un componente importante en los sistemas de agua dulce por su alta abundancia y diversidad, con relación a otros grupos biológicos. En estos sistemas acuáticos, muchos macroinvertebrados desarrollan gran parte de su ciclo de vida. Por lo anterior, el estudio de este grupo tiene gran importancia en temas de ecología de los sistemas acuáticos. El objetivo de este estudio fue caracterizar las comunidades de macroinvertebrados acuáticos asociados a la ciénaga Sahaya, en el departamento del Cesar y en tres de sus afluentes. En la ciénaga se recolectó el material biológico utilizando draga ekman y en los afluentes mediante red Surber. Se encontraron 6431 organismos, distribuidos 15 órdenes, 28 familias y 47 taxones. Del total de individuos, 5671 (88,2 \%), se recolectaron dentro de la ciénaga y los 760 individuos restantes (11,8 $\%$ ) en los afluentes evaluados. Se encontró que la familia con más abundancia fue Caenidae, con 1859 individuos, seguida por Naididae con 1691 y Chironomidae con 1122. La estructura de macroinvertebrados fue diferente en los cuerpos de agua corriente, de igual forma varió espacialmente entre las estaciones de la ciénaga y de los tres afluentes. En la ciénaga se evidenciaron posibles asociaciones entre la abundancia de algunos grupos de macroinvertebrados y las variables ambientales, hecho que parece confirmar el potencial de estas comunidades como indicadores de la calidad ecológica de las aguas.
\end{abstract}

PALABRAS CLAVE: Macroinvertebrados bentónicos, variables ambientales, ciénaga, quebradas, departamento del Cesar

\begin{abstract}
The aquatic macroinvertebrates represent an important biotic component in freshwater systems because they have high abundance and high diversity. In these systems, the macroinvertebrates develop large part of these life cycles. Therefore, macroinvertebrates are important for study of aquatic systems. The objective of this study was to describe the macroinvertebrate communities associated to Sahaya wetland and three tributaries. The biological samples were collected with dredge on wetland and Surber net on tributaries. 6431 organisms were collected, sorted in 15 orders, 28 families and 47 taxa. 5671 organisms (88.2\%) were collected on wetland and $760(11.8 \%)$ on the three tributaries. The families with high abundances were Caenidae (1859), Naididae (1691) and Chironomidae (1122). The structure of the community showed differences between the wetland and tributaries. In wetland, associations were evident between the higher abundance of some groups of macroinvertebrates with environmental variables. This confirms the potential of these communities as indicators of ecological water quality.
\end{abstract}

KEY WORDS: Aquatic macroinvertebrates, environmental variables, wetland, streams, departament of Cesar 


\section{INTRODUCCIÓN}

La comunidad bentónica es una de las más ricas y quizás de las más diversificadas en los sistemas acuáticos continentales (Esteves, 1988; Reinoso et al., 2008). Por tanto constituye un importante componente en la actividad metabólica de estos ambientes (Rivera et al., 2008; Montoya y Aguirre, 2009). Estos sistemas acuáticos están relacionados con los terrestres, los cuales aportan material que además de ser descompuesto por hongos y bacterias, también es utilizado por parte de los insectos acuáticos (Roldán y Ramírez, 2008). Esta acción de los organismos, acelera la descomposición del detrito, lo cual favorece al reciclaje de nutrientes (Rueda et al., 2006; Castellanos y Serrato, 2008). Los macroinvertebrados han adquirido especial importancia en el estudio de los sistemas dulceacuícolas debido a que actúan como testigos del nivel de deterioro ambiental de los sistemas lóticos y lénticos (Jaramillo, 2006). Además, por vivir en el sustrato, tienen un marcado carácter espacial y reflejan tanto las alteraciones del agua como las del propio sustrato, lo que los hace como elementos importantes para la evaluación biológica del agua (Calderón, 2004). Aunque en Colombia existen numerosos sistemas lénticos, los estudios sobre ellos han sido parciales, aislados e insuficientes, lo que quizás se debe a la falta de investigadores dedicados a este tipo de ecosistemas (Roldán y Ramírez, 2008; Pinilla-Agudelo, 2016). En el caso de los macroinvertebrados acuáticos, se evidencia una tendencia a investigar principalmente el papel de energía de esta comunidad en los ríos (Montoya y Aguirre, 2009; Zúñiga, 2010; Martínez-Rodríguez y Pinilla, 2014; Roldán et al., 2014). El desarrollo de inventarios faunísticos es una contribución importante para los sistemas lóticos de Colombia, debido a que son la etapa inicial de las evaluaciones de tipo ambiental, que se requiere conocer la composición y estructura de estas comunidades (Reinoso et al., 2008).

Para los cuerpos de agua lénticos en Colombia, desde hace una década, se ha documentado que el conocimiento de los macroinvertebrados a nivel de comunidades es muy escaso, en especial en los humedales de las zonas bajas (Duarte y Capador, 2006). Algunas aproximaciones son las de Amat et al. (2010), quienes exploraron algunos aspectos de la composición, riqueza y distribución de la entomofauna asociada a varias ciénagas del departamento de Córdoba. Otro trabajo para resaltar, es el realizado por MartínezRodríguez y Pinilla (2014), en el departamento del Cesar, donde evaluaron la calidad del agua de las ciénagas Zapatosa, Mata de Palma y La Pachita, mediante la caracterización de la comunidad de invertebrados acuáticos asociados a Eichhornia crassipes. El presente estudio se realizó con el fin de caracterizar la comunidad de macroinvertebrados bentónicos de la ciénaga de Sahaya y los caños Hato Viejo, Sucio y la quebrada Las Damas (municipio de Pelaya, Cesar). Además, se buscó analizar la relación de los organismos con algunas variables físicas y químicas de la ciénaga.

\section{MATERIALES Y MÉTODOS}

\section{Área de estudio}

La ciénaga de Sahaya presenta un área aproximada de $31,4 \mathrm{~km}^{2}$ y un perímetro medio de 37,89 km. Está influenciada por el río Magdalena y por los caños San Juan, Hato Viejo, Sucio y Orisco (CORPOCESAR, 2012). Se encuentra a $40 \mathrm{~m}$ de altitud. Según datos del IDEAM, la temperatura promedio multianual en la zona oscila entre 28,2 y $30,1{ }^{\circ} \mathrm{C}$ y los mayores valores se presentan de enero a abril y los menores de septiembre a diciembre. El régimen de lluvias es bimodal con dos periodos de precipitaciones menores entre los meses de abril y junio ( $31 \%$ de la precipitación anual), y precipitaciones mayores entre agosto y noviembre (53 \% de la precipitación anual). Los periodos secos comprenden de diciembre a marzo y julio (Jaramillo et al., 2013). Las lluvias anuales oscilan entre 1000 y 1400 mm (Rangel y Carvajal-Cogollo, 2013).

\section{Diseños y muestreos}

Se realizaron jornadas de recolecta mensual de macroinvertebrados acuáticos durante los meses de febrero, marzo, abril y julio de 2013, en cada sitio se tomaron tres réplicas, tanto en la ciénaga de Sahaya y como en los tributarios Hato Viejo, Sucio y quebrada Las Damas. El número de estaciones de muestreo estuvo asociada al tamaño del cauce de los tributarios, de esta manera se estableció una estación en Caño Hato Viejo (E1), dos en quebrada Las Damas (E2 y E3) y una en el Caño Sucio (E4). Las muestras se recolectaron lejos de la desembocadura para evitar la influencia del sistema léntico. En la ciénaga se seleccionaron seis estaciones (desde la E5 hasta la E10), de forma aleatoria a través de la construcción previa de una grilla (Figura 1), en cada sitio se realizaron tres réplicas. En la tabla 1 se describen de forma detallada los sitios de muestreo. 


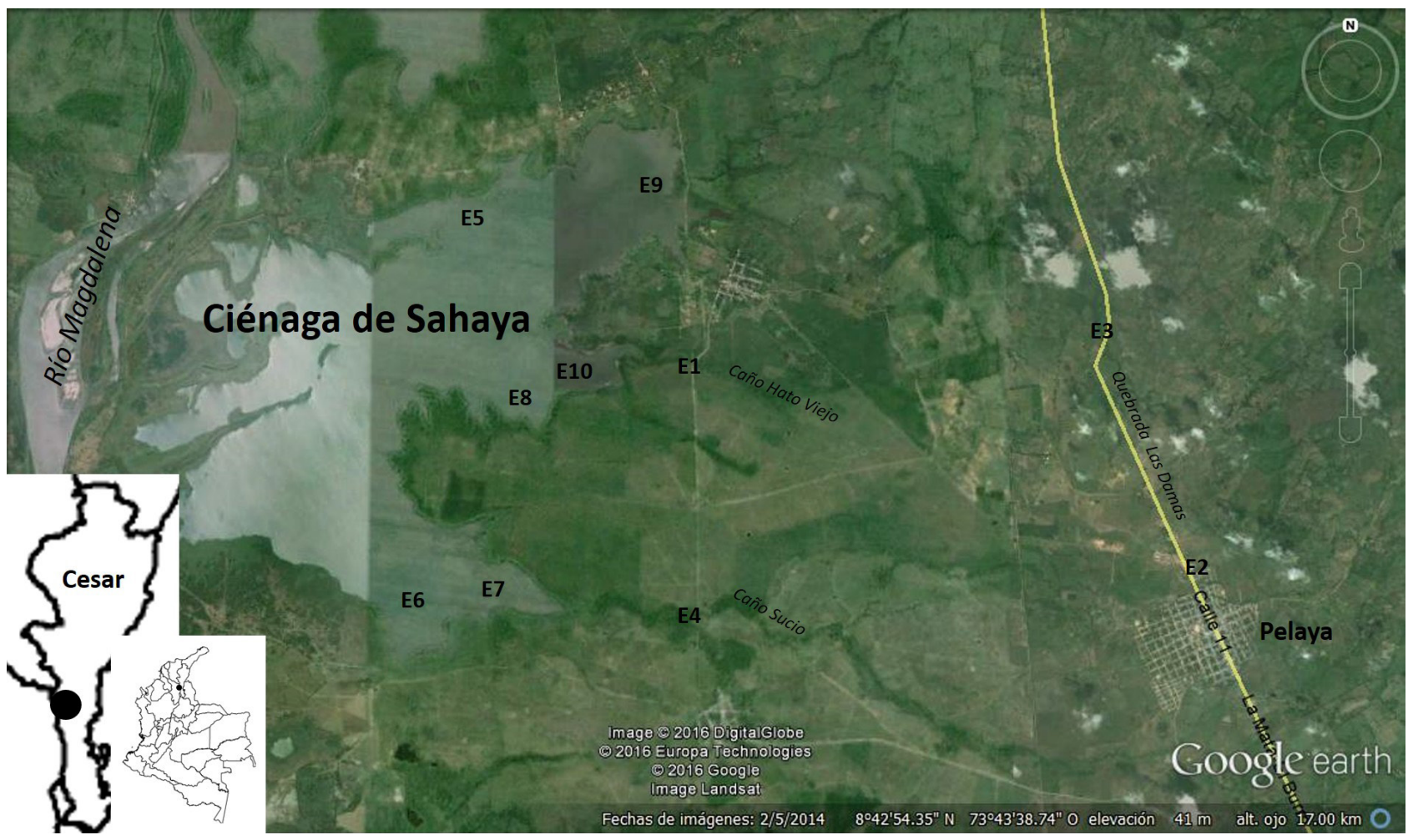

Figura 1. Localización y descripción de las estaciones de muestreo. E1 - E4: sitios en los efluentes y E5 - E10: sitios en la ciénaga.

Tabla 1. Localización geográfica, altitud y breve descripción de los sitios muestreados.

\begin{tabular}{|c|c|c|c|c|}
\hline \multirow{2}{*}{$\begin{array}{c}\text { Código de la } \\
\text { estación }\end{array}$} & \multicolumn{2}{|c|}{ Coordenadas } & \multirow{2}{*}{ Altitud (m) } & \multirow{2}{*}{ Descripción } \\
\hline & $\mathbf{N}$ & W & & \\
\hline E1 & $8^{\circ} 43,344^{\prime}$ & $73^{\circ} 43,746^{\prime}$ & 54 & $\begin{array}{l}\text { Aguas arriba del sitio se utilizaba como lavadero } \\
\text { doméstico de los campesinos del sector. Prediminio } \\
\text { de pastos y matorrales. }\end{array}$ \\
\hline E2 & $8^{\circ} 41,657^{\prime}$ & $73^{\circ} 40,610^{\prime}$ & 66 & $\begin{array}{l}\text { Esta es usada por los habitantes de Pelaya como } \\
\text { colector final de aguas servidas. }\end{array}$ \\
\hline E3 & $8^{\circ} 41,447^{\prime}$ & $73^{\circ} 43,718^{\prime}$ & 40 & $\begin{array}{l}\text { Presencia de monocultivo de palma de aceite. Algunas } \\
\text { fincas con árboles grandes, arbustos y matorrales. } \\
\text { Evidencia de algunas fuentes de aguas servidas. }\end{array}$ \\
\hline E4 & $8^{\circ} 44,761^{\prime}$ & $73^{\circ} 43,793^{\prime}$ & 51 & $\begin{array}{c}\text { Dominan árboles de gran porte. Pocas fincas } \\
\text { dedicadas a la ganadería. }\end{array}$ \\
\hline E5 & $8^{\circ} 44,183^{\prime}$ & $73^{\circ} 45,687^{\prime}$ & 38 & $\begin{array}{l}\text { Abundante vegetación alrededor del caño, también } \\
\text { se notó la presencia de macrófitas acuáticas. }\end{array}$ \\
\hline E6 & $8^{\circ} 44,480^{\prime}$ & $73^{\circ} 43,813^{\prime}$ & 42 & $\begin{array}{l}\text { Vegetación ribereña dominada por pastos y rastrojo. } \\
\text { Fuerte impacto de búfalos en el sector. }\end{array}$ \\
\hline E7 & $8^{\circ} 41,496^{\prime}$ & $73^{\circ} 44,732^{\prime}$ & 40 & Desembocadura de la Quebrada Las Damas. \\
\hline E8 & $8^{\circ} 42,843^{\prime}$ & $73^{\circ} 44,949$ & 42 & Vegetación ribereña dominante fue la palma de aceite. \\
\hline E9 & $8^{\circ} 44,599^{\prime}$ & $73^{\circ} 43,969^{\prime}$ & 43 & Desembocadura de la Quebrada Caño Sucio. \\
\hline E10 & $8^{\circ} 43,195^{\prime}$ & $73^{\circ} 44,312$ & 41 & Desembocadura del Caño Hato Viejo. \\
\hline
\end{tabular}




\section{Recolección y preservación de muestras}

Para la recolección de las muestras se utilizaron equipos diferentes, dependiendo el sistema evaluado. En la ciénaga se utilizó una draga Petersen de 18 x 22 cm y en los afluentes se usó una red Surber de $0,09 \mathrm{~m}^{2}$, con ojo de malla de $250 \mu \mathrm{m}$, teniendo en cuenta las recomendaciones de Zúñiga y Cardona (2009) y Ramírez (2010a). Las muestras se preservaron en alcohol al $96 \%$ en bolsas pláticas de calibre grueso debidamente etiquetadas. La identificación se realizó hasta el nivel más detallado posible mediante el uso de un estereoscopio Nikon SMZ745 y las claves taxonómicas de Ruíz et al. (2000); Martínez et al. (2003); Merritt et al. (2008); Domínguez y Fernández (2009); Ramírez (2010b) y Springer (2010).

\section{Análisis físicos y químicos}

En el campo se midió el oxígeno disuelto (mg/L), el $\mathrm{pH}$ (unidades) y la temperatura $\left({ }^{\circ} \mathrm{C}\right)$ empleando una sonda multiparamétrica (WTW 340i). En cada estación se tomó una muestra de agua para su posterior análisis en el laboratorio, donde se midió la Turbidez (NTU) y la dureza total $\left(\mathrm{mg} / \mathrm{L} \mathrm{CaCO}_{3}\right)$ a través de métodos colorimétricos estandarizados (APHA, 1992).

\section{Análisis de datos}

Para determinar el grado de asociación entre las comunidades de macroinvertebrados de las diez estaciones de muestreo, se utilizó el índice de similitud de Jaccard. Con la matriz resultante, se realizó un análisis de ordenamiento por escalamiento multidimensional no métrico (nMDS, por sus siglas en inglés), con el fin de determinar posibles relaciones en la distribución espacial de los organismos entre estaciones de la ciénaga y los cuerpos de agua. También se realizó un NPMANOVA, para evaluar la significancia del patrón generado en el nMDS (teniendo en cuenta los taxones más abundantes). Por último se hizo un Análisis de Correspondencia Canónica (ACC), para determinar la posible asociación entre los macroinvertebrados acuáticos de la ciénaga y las variables físicas y químicas, previa estandarización de los datos. Los análisis se realizaron en el programa libre R (www.r-project.com).

\section{RESULTADOS}

\section{Caracterización ambiental de la ciénaga}

Las variables ambientales en la ciénaga no presentaron diferencias espaciales (NPMANOVA p >0,05), pero si se observaron variaciones entre las estaciones. En E7 y E8 se registraron los valores más bajos de oxígeno disuelto $(4,93$ y $4,65 \mathrm{mg} / \mathrm{L})$ y de $\mathrm{pH}(7,78$ y 8,00$)$ respectivamente. En E6 la turbidez fue la variable de menor valor (102,30 NTU) y en las estaciones E5 y E10 predominó la alta turbidez (144,67 y 104,67 NTU); en E10 se detectó la mayor temperatura promedio del agua $\left(32,03{ }^{\circ} \mathrm{C}\right)$. Finalmente en E6 y E9 se evidenciaron los valores más altos de oxígeno disuelto $(6,60$ y 5,85 $\mathrm{mg} / \mathrm{L}$ ) (Tabla 2).

\section{Composición faunística}

Se registraron 6429 macroinvertebrados bentónicos distribuidos en siete clases, 15 órdenes, 28 familias y 42 taxones en todas las estaciones (afluentes y ciénaga). Del total de individuos 5669 (88,2 \%) se recolectaron en la ciénaga y 760 (11,8 \%) en los afluentes. En términos generales, Ephemeroptera presentó la mayor abundancia (36,30 \%), seguido por Haplotaxida (33,02 \%) y Diptera $(23,67 \%)$. Mientras que los grupos menos abundantes fueron Architaenioglossa (5,31 \%) y Odonata (1,70 \%). La familia, Caenidae (Ephemeroptera) fue la más abundante con 1859 individuos, seguida por Naididae (Haplotaxida) 1699, Chironomidae (Diptera) 1122, Polymitarcyidae (Ephemeroptera) 505, Ceratopogonidae (Diptera) 357 y Elmidae (Coleoptera) 329 individuos. En conjunto estas seis familias aportaron cerca del 80 $\%$ de la abundancia total (Tabla 3).

En este trabajo se destaca la presencia de los géneros Brasisliocaensis y Campsurus (Ephemeroptera) Disersus (Coleoptera) y Pisidium (Bivalvia), los cuales son los primeros registros de macroinvertebrados en ciénagas de tierras bajas del Caribe colombiano. 
Tabla 2. Variables físicas y químicas evaluadas durante los muestreos en las seis estaciones de la ciénaga de Sahaya.

\begin{tabular}{lrrrrrr}
\hline & \multicolumn{7}{c}{ Estación de muestreo } \\
\cline { 2 - 6 } Variable ambiental & E5 & E6 & E7 & E8 & E9 & E10 \\
\hline Temperatura $\left({ }^{\circ} \mathrm{C}\right)$ & 30,23 & 31,57 & 31,83 & 31,87 & 31,77 & 32,03 \\
Oxígeno disuelto (mg/L) & 5,72 & 6,60 & 4,93 & 4,65 & 5,85 & 5,13 \\
pH (unidades) & 8,11 & 8,31 & 7,78 & 8,00 & 8,05 & 8,20 \\
Turbiedad (NTU) & 144,67 & 77,27 & 88,57 & 90,13 & 96,60 & 104,67 \\
Dureza total (mg/L) & 80,30 & 102,30 & 74,57 & 77,87 & 96,77 & 76,83 \\
\hline
\end{tabular}

\section{Distribución de los macroinvertebrados y su relación con las variables físicas y químicas}

Basados en una matriz de presencia-ausencia de cada taxón en las diez estaciones, se realizó un análisis de agrupamiento para determinar la asociación entre la ciénaga y los cuerpo de aguas corrientes, en el nMDS se observa que las comunidades se organizaron de acuerdo al tipo de sistema (léntico o lótico). Las diez estaciones evaluadas conformaron dos grandes grupos, el primero constituido por las seis estaciones de la ciénaga de Sahaya (E6 - E10), donde E5 conforma un subgrupo y el segundo grupo conformado por las cuatro estaciones (E1 - E4) de la Quebrada Las Damas, el Caño Sucio y el caño Hato Viejo, E3 constituye un subgrupo dentro los sistemas lóticos (Figura 2).

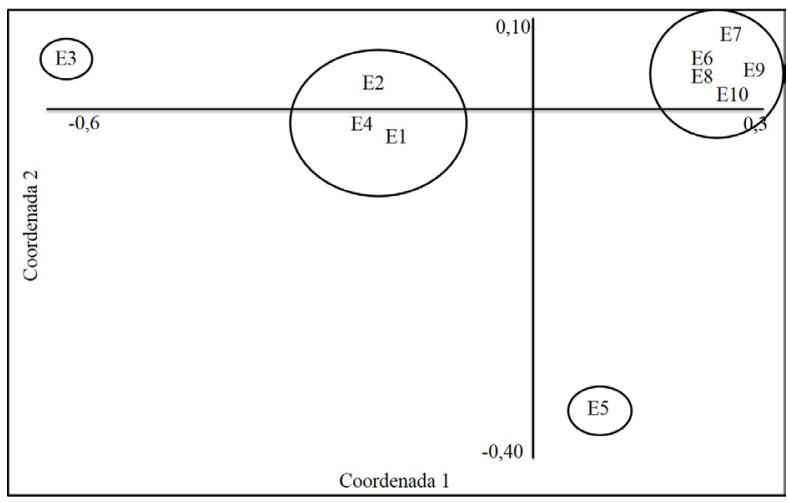

Figura 2. Análisis de ordenamiento nMDS de las estaciones de muestreo basado en las abundancias en los afluentes (E1 - E4) y la ciénaga (E5 - E10). La clasificación se realizó al 50 \% de similitud. Los datos fueron trasformados a $\log (X+1)$ Stress: 0,04.
La estación San Bernardino (E5) compartió menos del $50 \%$ de macroinvertebrados, con las demás estaciones de la Ciénaga de Sahaya. Las estaciones E6 - E10, conformaron un grupo en el que comparten por lo menos el $50 \%$ de los taxones de macroinvertebrados. Del mismo modo, la estación Caño Sucio (E3), de los sistemas lóticos, comparten menos del $50 \%$ de su composición biótica con las otras estaciones.

El patrón espacial de los macroinvertebrados muestra un gradiente en el que claramente se separan hacia el extremo derecho las estaciones de la ciénaga. En esos sitios estuvieron presente las familias Naididae, Ceratopogonidae, Chironomidae y Ampullariidae. Al extremo izquierdo se pueden observar las estaciones de cuerpos de aguas corrientes, en donde se presentaron los taxones Caenidae, Corduliidae y Libellulidae. En concordancia, la prueba NPMANOVA evidenció que la estructura de macroinvertebrados en las estaciones de los dos grandes grupos presentaron diferencias significativas $(\mathrm{p}<0,05)$.

En el ACC la varianza explicada por los dos primeros ejes canónicos fue del 87,92 \%. Este análisis sugiere que los organismos de la familia Naididae parecen tener una tendencia a aumentar cuando disminuye la Turbidez. Mientras que los individuos de los taxones Bullinilidae y Orthocladiinae, mostraron tendencia a relacionarse de manera directa con la turbidez. Los organismos de la familia Ceratopogonidae parecen asociarse a altos valores de $\mathrm{pH}$ y Temperatura. Del mismo modo, Hydrobiidae parece asociarse con los altos niveles de oxígeno. Las mayores abundancias de Campsurus se asociaron con bajos valores de oxigeno y dureza total (Figura 3). 


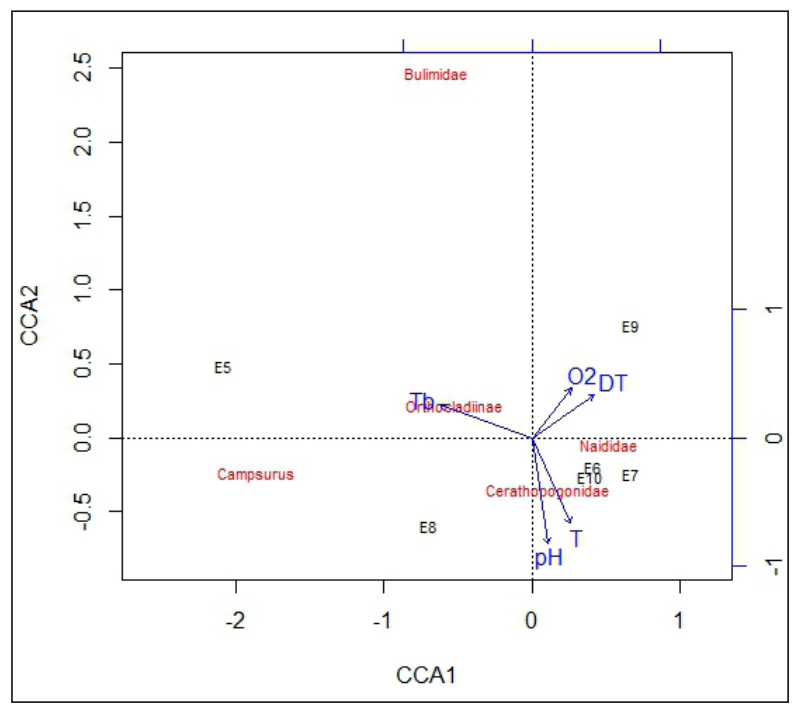

Figura 3. Análisis de correspondencia canónica (ACC) mostrando la relación existente entre los taxones de macroinvertebrados bentónicos de los sitios de muestreo de la ciénaga de Sahaya y las variables oxígeno disuelto $\left(\mathrm{O}_{2}\right), \mathrm{pH}$, dureza total (DT), temperatura (T), y turbiedad (Tb).

\section{DISCUSIÓN}

Es común que en los sistemas lénticos tropicales se presente un alto déficit de oxígeno (Lewis, 2000). Por lo tanto, se considera que los valores de oxígeno disuelto detectados en las seis estaciones de la ciénaga de Sahaya, correspondan a sistemas típicos de esta zona [entre 4,5 y 8,5 unidades, según Roldán (2003); Roldán y Ramírez (2008)]. Los registros de $\mathrm{pH}$ se encuentran dentro de los límites de supervivencia de los organismos acuáticos, entre 4,5 y 8,5 unidades, según Roldán y Ramírez (2008). A pesar de estas condiciones de oxígeno y pH, la ciénaga de Sahaya se encuentra rodeada por grandes extensiones de monocultivos como la palma de aceite y está influenciada por la ganadería extensiva, cuya actividad puede provocar por arrastre la adición de sedimento y otros elementos al sistema, lo que explicarían en principio los altos valores de turbidez.

Los órdenes de macroinvertebrados que presentaron mayores abundancias en este trabajo (Unionoida, Haplotaxida y Diptera) poseen requerimientos ecológicos que pueden relacionarse con ciénagas de tierras bajas con alta tasa de sedimentación. En este sentido Unionoida pertenece a uno de los pocos grupos de moluscos bivalvos que han tenido radiación exitosa y significativa en ambientes acuáticos continentales (Domínguez y Fernández, 2009; Zúñiga y Cardona, 2009; Roldán et al., 2014). El orden Diptera contienen más especies dulceacuícolas que cualquier otro grupo de macroinvertebrados acuáticos y son capaces de colonizar muchos tipos de hábitats (Restrepo y Rincón, 2009; Martínez-Rodríguez y Pinilla, 2014; Roldán et al., 2014). La mayoría de los Haplotaxida parecen tener exclusividad hacia los fondos lodosos de sistemas eutrofizados con abundante materia orgánica en descomposición (Roldán, 2003; Roldán y Ramírez, 2008; Zúñiga y Cardona, 2009), condiciones que seguramente favorecieron a este grupo, y que se reflejaron con sus altas abundancias de la ciénaga de Sahaya. Roldán (2003) afirma que los oligoquetos se encuentran asociados a bajas concentraciones de oxígeno, gas que se reduce aún más cuando la temperatura del agua es alta, como ocurre en la ciénaga estudiada.

En comparación con estudios similares, Deluque et al. (2006), registraron 20 familias en la ciénaga de San Antonio, Martínez-Rodríguez y Pinilla (2014), 32 familias en las ciénagas Zapatosa, Mata de Palma y La Pachita y Rúa (2015), 25 familias en la ciénaga de Zapayán. Aunque en este trabajo solo se encontraron 15 familias en la ciénaga se Sahaya, esto se debe a que los demás trabajos analizaron las raíces de macrófitas, las cuales ofrecen mejores condiciones de heterogeneidad de microhábitats que favorecen el establecimiento de un mayor número de taxones (Martínez-Rodríguez, 2014), mientras que en este estudio solo se analizaron muestras del fondo, las cuales usualmente tienen menor complejidad estructural (Junk y Wantzen, 2004).

En general, la distribución siguió el patrón esperado, la ubicación del sitio E5 fuera del grupo de las estaciones de la ciénaga, pudo deberse a la mayor abundancia de las macrófitas y la presencia de un bosque ribereño asociado a una menor presión antrópica en este lugar, a diferencia de las otras estaciones. Estos resultados coinciden con lo registrado por Reyes (2013), quien encontró que en estaciones de sistemas lénticos con cobertura boscosa se presentaron mayor abundancia y diversidad de taxones, debido a que tienen una mayor disponibilidad de sustratos. Igualmente este autor afirma que las macrófitas proveen una gran oferta de recursos alimenticios. Por otro lado, el grupo conformado por las estaciones E6 a E10 estuvieron influenciadas por la desembocadura de los caños Hato Viejo, Sucio y Quebrada Las Damas, lo cual pudo generar una influencia hidrológica parecida en estos sitios, lo que pudo producir una composición similar de las comunidades (Deluque et al., 2006). Sin embargo, se requieren trabajos experimentales que comprueben dicha hipótesis. 
Tabla 3. Abundancia de los macroinvertebrados encontrados en cada sitio de trabajo. E: estaciones de muestreo.

\begin{tabular}{|c|c|c|c|c|c|c|c|c|c|c|c|c|c|c|}
\hline \multirow{3}{*}{ CLASE } & \multirow{3}{*}{ ORDEN } & \multirow{3}{*}{ FAMILIA } & \multirow{3}{*}{ SUBFAMILIA } & \multirow{3}{*}{ TAXÓN } & \multicolumn{10}{|c|}{ Estaciones } \\
\hline & & & & & \multicolumn{4}{|c|}{ Quebradas / Caños } & \multicolumn{6}{|c|}{ Ciénaga Sahaya } \\
\hline & & & & & E1 & E2 & E3 & E4 & E5 & E6 & E7 & E8 & E9 & E10 \\
\hline Clitellata & Haplotaxida & Naididae & Naidinae & $\begin{array}{l}\text { Morfo } \\
\text { Naididae }\end{array}$ & 46 & 48 & 21 & 28 & 38 & 253 & 191 & 208 & 402 & 464 \\
\hline Arachnida & Trombidiforme & Hydrachnidae & & $\begin{array}{l}\text { Morfo } \\
\text { Hydrachnidae }\end{array}$ & 2 & 0 & 0 & 0 & 1 & 0 & 0 & 0 & 0 & 0 \\
\hline \multirow[t]{4}{*}{ Crustáceo } & Decapoda & Trichodactylidae & Dilocarcininae & Sylviocarcinus & 0 & 0 & 3 & 0 & 0 & 0 & 0 & 0 & 0 & 0 \\
\hline & Stylomatophora & Bullinidae & & Morfo Bullinidae & 0 & 0 & 0 & 0 & 29 & 8 & 0 & 0 & 42 & 1 \\
\hline & & Ancylidae & & Morfo Ancylidae & 0 & 0 & 1 & 1 & 0 & 0 & 0 & 0 & 0 & 0 \\
\hline & & & & $\begin{array}{l}\text { Morfo } \\
\text { Ampullariidae }\end{array}$ & 1 & 2 & 0 & 0 & 0 & 0 & 0 & 0 & 0 & 0 \\
\hline \multirow[t]{5}{*}{ Gastropoda } & & Ampullariidae & & Pomacea & 0 & 2 & 0 & 0 & 0 & 0 & 0 & 0 & 0 & 0 \\
\hline & Basommathophora & & & Marisa & 0 & 0 & 0 & 0 & 0 & 0 & 0 & 1 & 0 & 0 \\
\hline & & Ampullaridae & & cf. Biomphalaria & 0 & 0 & 1 & 0 & 0 & 0 & 0 & 0 & 0 & 0 \\
\hline & & Hydrobiidae & & $\begin{array}{l}\text { Morfo } \\
\text { Hydrobiidae }\end{array}$ & 0 & 0 & 6 & 3 & 14 & 3 & 0 & 1 & 0 & 6 \\
\hline & Veneroida & Sphaeriidae & & Pisidium & 0 & 0 & 3 & 0 & 29 & 0 & 0 & 0 & 7 & 0 \\
\hline \multirow[t]{7}{*}{ Bivalvia } & & Unionidae & & Morfo Unionidae & 0 & 20 & 0 & 0 & 6 & 0 & 38 & 1 & 0 & 0 \\
\hline & Unionoida & Mycetopodidae & & $\begin{array}{l}\text { Morfo } \\
\text { Mycetopodidae }\end{array}$ & 0 & 0 & 0 & 0 & 27 & 0 & 2 & 0 & 14 & 0 \\
\hline & & Belastomatidae & Heterocorixinae & Heterocorixa & 1 & 0 & 3 & 0 & 0 & 0 & 0 & 0 & 0 & 0 \\
\hline & & & Belostomatinae & Belostoma & 3 & 2 & 0 & 2 & 0 & 0 & 0 & 0 & 0 & 0 \\
\hline & Hemiptera & Notonectidae & Anisopinae & Buenoa & 0 & 0 & 0 & 8 & 0 & 0 & 0 & 0 & 0 & 0 \\
\hline & & & Notonectinae & Notonecta & 1 & 0 & 0 & 0 & 0 & 0 & 0 & 0 & 0 & 0 \\
\hline & & Naucoridae & Cryphocricinae & Cryphocricos & 1 & 0 & 0 & 0 & 0 & 0 & 0 & 0 & 0 & 0 \\
\hline \multirow{8}{*}{ Insecta } & & Tipulidae & Limnophilinae & Hexatoma & 0 & 0 & 3 & 0 & 0 & 0 & 0 & 0 & 0 & 0 \\
\hline & & Cerathopogonidae & & $\begin{array}{l}\text { Morfo } \\
\text { Ceratopogonidae }\end{array}$ & 14 & 99 & 8 & 67 & 15 & 71 & 14 & 28 & 23 & 18 \\
\hline & & Culicidae & & Morfo Culicidae & 2 & 0 & 0 & 0 & 0 & 0 & 0 & 0 & 0 & 0 \\
\hline & Diptera & & Tanypodinae & $\begin{array}{l}\text { Morfo } \\
\text { Tanypodinae }\end{array}$ & 10 & 12 & 23 & 5 & 12 & 0 & 3 & 4 & 0 & 1 \\
\hline & & Chironomidae & Orthocladiinae & $\begin{array}{l}\text { Morfo } \\
\text { Orthocladiinae }\end{array}$ & 38 & 25 & 182 & 69 & 29 & 23 & 12 & 9 & 13 & 23 \\
\hline & & & Chironominae & $\begin{array}{l}\text { Morfo } \\
\text { Chironominae }\end{array}$ & 136 & 265 & 89 & 106 & 4 & 7 & 4 & 6 & 8 & 4 \\
\hline & Fubamorontor & Caenidae & & Brasilocaenis & 55 & 115 & 1524 & 162 & 0 & 0 & 0 & 0 & 0 & 3 \\
\hline & & Polymitarcyidae & & Campsurus & 55 & 2 & 16 & 37 & 167 & 21 & 7 & 139 & 1 & 60 \\
\hline
\end{tabular}

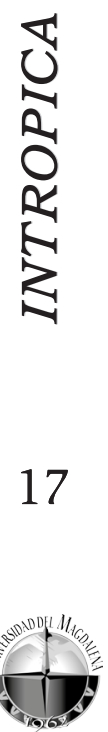


Continuación Tabla 3.

\begin{tabular}{|c|c|c|c|c|c|c|c|c|c|c|c|c|c|c|}
\hline \multirow{3}{*}{ CLASE } & \multirow{3}{*}{ ORDEN } & \multirow{3}{*}{ FAMILIA } & \multirow{3}{*}{ SUBFAMILIA } & \multirow{3}{*}{ TAXÓN } & \multicolumn{10}{|c|}{ Estaciones } \\
\hline & & & & & \multicolumn{4}{|c|}{ Quebradas / Caños } & \multicolumn{6}{|c|}{ Ciénaga Sahaya } \\
\hline & & & & & E1 & E2 & E3 & $\mathrm{E} 4$ & E5 & E6 & E7 & E8 & E9 & E10 \\
\hline \multirow{16}{*}{ Insecta } & Trichoptera & Polycentropodidae & Polycentropodinae & Polycentropus & 4 & 0 & 0 & 5 & 0 & 0 & 0 & 0 & 0 & 0 \\
\hline & \multirow{9}{*}{ Coleoptera } & Psephenidae & & Psephenus & 11 & 1 & 11 & 27 & 0 & 0 & 0 & 0 & 0 & 0 \\
\hline & & \multirow{2}{*}{ Elmidae } & & Dysersus & 89 & 38 & 14 & 150 & 0 & 0 & 1 & 0 & 14 & 3 \\
\hline & & & & Cylloepus & 6 & 0 & 8 & 6 & 0 & 0 & 0 & 0 & 0 & 0 \\
\hline & & Noteridae & & Morfo Noteridae & 2 & 0 & 0 & 0 & 0 & 0 & 0 & 0 & 0 & 0 \\
\hline & & Georissidae & & Georissus & 10 & 0 & 0 & 6 & 0 & 0 & 0 & 0 & 0 & 0 \\
\hline & & \multirow{4}{*}{ Hidrophilidae } & Hidrophilinae & Hydrophilus & 3 & 0 & 0 & 0 & 0 & 0 & 0 & 0 & 0 & 0 \\
\hline & & & Acidocerinae & Helochares & 1 & 0 & 0 & 0 & 0 & 0 & 0 & 0 & 0 & 0 \\
\hline & & & & $\begin{array}{l}\text { Morfo } \\
\text { Hydrophilidae }\end{array}$ & 0 & 4 & 0 & 0 & 0 & 0 & 0 & 0 & 0 & 0 \\
\hline & & & Hidrophilinae & Berosus & 19 & 0 & 1 & 35 & 0 & 0 & 0 & 0 & 0 & 0 \\
\hline & \multirow{6}{*}{ Odonata } & Libellulidae & Corduliineae & Morfo Corduliinae & 13 & 3 & 1 & 11 & 0 & 0 & 0 & 0 & 0 & 0 \\
\hline & & Protoneuridae & & $\begin{array}{l}\text { Morfo } \\
\text { Protoneuridae }\end{array}$ & 0 & 0 & 23 & 0 & 0 & 0 & 0 & 0 & 0 & 0 \\
\hline & & & & Aphylla & 0 & 0 & 0 & 1 & 0 & 0 & 0 & 0 & 0 & 0 \\
\hline & & Gonphidae & & Phyllogomphoides & 1 & 2 & 0 & 2 & 0 & 0 & 0 & 0 & 0 & 0 \\
\hline & & & & Epigomphus & 1 & 0 & 0 & 0 & 0 & 0 & 0 & 0 & 0 & 0 \\
\hline & & Libellulidae & Libellulinae & Morfo Libellulinae & 7 & 36 & 15 & 1 & 0 & 0 & 0 & 0 & 0 & 0 \\
\hline
\end{tabular}

En cuanto a los afluentes, la estación E3 se separó de las otras estaciones lóticas. En dicho sitio se presentó una mejor cobertura boscosa asociada en la ribera y fue la más distante de los centros poblados. El agrupamiento de las estaciones E1, E2 y E4, pudo deberse a que las corrientes presentaron incidencia antrópica en la mayor parte de su cauce, representada en una menor densidad y altura de la vegetación ribereña. La degradación de esta vegetación pudo afectar la disponibilidad de microhábitats en el sistema y por ende pudo influir en que se diera una estructura de la comunidad de los macroinvertebrados (Restrepo y Rincón, 2009).

El aumento de la turbidez del agua puede deberse al incremento de la materia orgánica e inorgánica 18 suspendidas, tanto particulada fina como particulada gruesa. Otra causa es la presencia de materiales en forma coloidal, como la arcilla (Roldán y Ramírez, 2008). Al respecto, Martínez-Rodríguez y Pinilla (2014), señalan que una ciénaga natural contiene de forma natural mucha materia orgánica. No es de extrañarse entonces la asociación con la turbidez que mostraron los taxones: Bullinidae, Hydrobiidae, Mycetopodidae, Pisidium y Tanypodinae. Por su parte, los quironómidos de la subfamilia Tanypodinae poseen una gran capacidad de adaptación a las condiciones que el medio les ofrece, por lo que se les suele encontrar en hábitats muy diversos (Ruíz et al., 2000). Por otro lado, los organismos del género Pisidium, habitan en ambientes muy sedimentados y casi anóxicos, donde pocos bivalvos puede vivir (Valdovinos, 2008). Los moluscos de la familia Mycetopodidae prefieren aguas poco profundas con fondos arcillosos y arenosos (arena fina) con materia orgánica de origen vegetal en descomposición (Martínez et al., 2003). El género Heleobia y la familia Bullinidae, pertenecientes a la clase Gasteropoda, se caracterizan por vivir en medios con abundante materia orgánica en descomposición (Romero, 2015).

En síntesis, la ciénaga presentó alta turbidez, debido probablemente a una gran cantidad de materia particulada en suspensión. Los taxones como Orthocladiinae, 
Bullinidae, Hydrobiidae, Tanypodinae, Pisidium y Mycetopodidae mostraron afinidad con esa alta turbidez, y esto se evidenció en una distribución uniforme de las comunidades de macroinvertebrados en los sitios de la ciénaga con mayor turbidez. Al parecer, las comunidades de macoinvertebrados responden a cambios en el ambiente, tanto en el ambiente léntico, como en los sistemas lóticos.

\section{AGRADECIMIENTOS}

A la Fundación Hidrobiológica George Dahl por financiar esta investigación. Al Grupo de Investigación en Biodiversidad y Ecología Aplicada (GIBEA) de la Universidad del Magdalena. A los evaluadores anónimos por sus importantes aportes al documento.

\section{BIBLIOGRAFÍA}

Amat, G., Fernández, F., Jiménez, L., Morales, I., Rojas, N. y Devia, N. 2010. Entomofauna terrestre y semiacuática de las ciénagas de los ríos Sinú y San Jorge (Departamento de Córdoba, Colombia). En: Rangel, J., Editor. Colombia Diversidad biótica IX. Ciénagas de Córdoba: Biodiversidad ecológica y manejo ambiental. Santa Fe de Bogotá, D. C. Colombia.

APHA. 1992. Standard methods for the examination of water and waste water. American Public Health Association, New York.

Calderón, J. 2004. Evaluación de la comunidad de macroinvertebrados bentónicos y la calidad fisicoquímica del agua en la parte alta de la quebrada el Carracá del Municipio de los Santos departamento Santander. Monografía para Especialización, Universidad Industrial de Santander, Bucaramanga, Santander, Colombia.

Castellanos, P. y Serrato, C. 2008. Diversidad de macroinvertebrados acuáticos en un nacimiento de río en el Páramo de Santurbán, Norte de Santander. Revista Académica Colombiana de Ciencias 32(122): 79-86.

CORPOCESAR - Corporación Autónoma Regional del Cesar. 2012. Resolución No.0720. Resoluciones._URL:_http://www. corpocesar.gov.co/normas.html. Consultado: 2 de Agosto 2015.

Deluque, J., Reyes, S., Sierra-Labastidas, T. y López, W. 2006. Primeros reportes de familias de macroinvertebrados asociados a macrófitas acuáticas en la ciénaga del Cerro de San Antonio (Río Magdalena, Colombia). Revista Intropica 3: 77-86.

Domínguez, E. y Fernández, R. 2009. Macroinvertebrados Bentónicos Sudamericanos. Sistemática y Biología. Fundación Miguel Lillo, Tucumán, Argentina.
Esteves, F. 1988. Fundamentos de limnología. Editora Interciencia/Finep, Rio de Janeiro.

Duarte, Y. y Capador, R. 2006. Entomofauna acuática asociadada a Paspalum repens (Poacea) en los lagos I y IV del sistema lagunar Yahuarcaca (Leticia - Amazonas). Tesis de pregrado, Universidad Pedagógica Nacional, Facultad de Ciencia y tecnología, Departamento de Biología, Bogotá, D.C.

Jaramillo, J. 2006. Estudio de las comunidades de macroinvertebrados en el área del embalse de Ponce II y su relación con la calidad del agua. Revista de Ingenierías Universidad de Medellín 5(8): 45-58.

Jaramillo, A., Villamizar. V., Calvo, J., Rangel, O. y Parra, N. 2013. Los sedimentos de las ciénagas el Congo, Musanda, Doña María, El Sordo, Juncal, Baquero, Morales y Costilla. En: Rangel, J., Editor. Colombia diversidad biótica XIII, Complejo cenagoso Zapatosa y ciénagas del sur del Cesar. Santa Fe de Bogotá D.C. Colombia.

Junk, W. y Wantzen, K. 2004. The flood pulse concept: New aspects, approaches and applications - An update. En: Welcomme, R.L. y Petr, T., Editores. Proceedings of the second international symposium on the management of large rivers for fisheries. Vol. 2. Food and Agricultural Organization and Mekong River Commision. FAO Regional Office for Asiaand Pacific, Bangkok.

Lewis, M. 2000. Basis for the protection and management of tropical lakes. Lakes and Reservoirs: Research and Management 5: 35-48.

Martínez, R. Cabrera, A. y Lasso, C. 2003. Moluscos bivalvos (Unionacea y Mutelacea) de la cuenca del río Orinoco, Venezuela. Memoria de la Fundación La Salle de Ciencias Naturales 159-160: 283-303.

Martínez-Rodríguez, M. y Pinilla, G.A. 2014. Valoración de la calidad del agua de tres ciénagas del departamento del Cesar mediante macroinvertebrados asociados a Eichhornia crassipes (Pontederiaceae). Revista Caldasia 36(2): 305-321.

Merritt, R.W., Cummins, K.W. y Berg, M.B. 2008. An introduction to the aquatic insects of North America. 4th ed. Kendall/Hunt: Iowa, EEUU.

Montoya, Y. y Aguirre, N. 2009. Estado del arte de la limnología de lagos de planos inundables (Ciénagas) en Colombia. Revista Gestión y Ambiente 12(3): 85-106.

Pinilla-Agudelo, G.A. 2016. Algunas experiencias en el uso de índices limnológicos en Colombia. Acta Biológica Colombiana 21(1)Supl: S241-S248. doi: http://dx.doi. org/10.15446/abc.v21n1sup.51073 
Ramírez, A. 2010a. Métodos de recolección. Revista de Biología Tropical 58(Suppl 4): 41-50.

Ramírez, A. 2010b. Odonata. Revista de Biología Tropical 58(Suppl 4): 97-136.

Rangel, J. y Carvajal-Cogollo, J. 2013. Clima de los alrededores de las ciénagas del centro y del sur del departamento del Cesar. En: Rangel, J., Editor. Colombia diversidad biótica XIII Complejo cenagoso Zapatosa y ciénagas del sur del Cesar. Biodiversidad, conservación y manejo. Santa Fe de Bogotá D.C. Colombia.

Reinoso, G., Gutiérrez, C., López, E., Carranza, X. y Vásquez, J. 2008. Macroinvertebrados acuáticos. En: Reinoso, G., Navarro, F., Melo, J., Vejarano, M. y Esquivel, H., Editores. Biodiversidad faunística y florística de la subcuenca del río Anamichú, (Cuenca del rio Saldaña), Biodiversidad Regional Fase IV. Universidad del Tolima, Ibagué, Colombia.

Restrepo, M. y Rincón, C. 2009. Aproximación a la caracterización ecológica de la quebrada Fucha en la reserva forestal de los cerros orientales en la localidad de San Cristóbal. (Bogotá Colombia). Revista Bio-investigación 2: 1-17.

Reyes, F. 2013. Macroinvertebrados acuáticos de los cuerpos lénticos de la región Maya, Guatemala. Revista Científica 23(1): 7-16.

Rivera, J., Camacho, D. y Votero, A. 2008. Estructura numérica de la entomofauna acuática en ocho quebradas del departamento del Quindío-Colombia. Acta Biológica Colombiana 13(2): 133-146.

Roldán, G. 2003. Bioindicación de la calidad del agua en Colombia. Uso del método BMWP/Col. Universidad de Antioquia, Medellín, Antioquia, Colombia.

Roldán, G. y Ramírez, J.J. 2008. Fundamentos de limnología tropical. Universidad de Antioquia, Universidad Católica de Oriente, Academia Colombiana de Ciencias Exactas, Físicas y Naturales, Medellín, Antioquia, Colombia.

Roldán, G., Zúñiga, M. del C., Zamora, H., Reinoso, G., Álvarez, L.F. y Longo, M. 2014. Colombia. En: Alonso-
EguíaLis, P., Mora, J.M., Campbell, B. y Springer, M., Editores. Diversidad, conservación y uso de los macroinvertebrados dulceacuícolas de México, Centroamérica, Colombia, Cuba y Puerto Rico. Instituto Mexicano de Tecnología del Agua y Universidad Autónoma de Querétano, México y Universidad de Costa Rica, San José-Costa Rica.

Romero, L. 2015. Diagnóstico hidrobiológico (macrófitas y macroinvertebrados acuáticos) para el humedal urbano panamericano en el Valle del Cauca, como insumo para la formulación de un plan de manejo. Tesis de pregrado, Universidad Autónoma de Occidente, Santiago de Cali, Valle del Cauca, Colombia.

Rúa, G. 2015. Macroinvertebrados acuáticos asociados a raíces de Eichhornia crassipes (Mart) Solms, en la ciénaga de Zapayán, Magdalena-Colombia. Revista Intropica 10: 52-59.

Rueda, G., Wantzen, K.M. y Beltrán, L.M. 2006. Leaf-litter decomposition in an Amazonian floodplain stream: effects of seasonal hydrological changes. Journal North American Benthological Society 25(1): 231-247.

Ruíz, J., Ospina, R., Gómez, H. y Riss, W. 2000. Guía para la identificación genérica de larvas de quironómidos (Diptera: Chironomidae) de la sabana de Bogotá. III. subfamilias Tanypodinae, Podonominae y Diamesinae. Caldasia 22(1): 34-60.

Springer, M. 2010. Trichoptera. Revista de Biología Tropical 58(Suppl 4): 151-198.

Valdovinos, C. 2008. Invertebrados dulceacuícolas En: Rovira, J., Ugalde, J. y Stutzin, M., Editores. Biodiversidad de Chile. Patrimonios y desafíos. Conama, Chile.

Zúñiga, M. del C. 2010. Diversidad, distribución y ecología del Orden Plecoptera (Insecta) en Colombia, con énfasis en Anacroneuria (Perlidae). Momentos de Ciencia 7(2): 101-112.

Zúñiga, M. del C. y Cardona, W. 2009. Bioindicadores de la Calidad de Agua y Caudal Ambiental. En: Cantera, J., Carvajal, J. y Castro, L.M., Editores. Caudal Ambiental: Conceptos, Experiencias y Desafíos. Programa Editorial de la Universidad del Valle, Colección Libros de Investigación. Cali, Colombia. 